\title{
Emicizumab in the Treatment of Acquired Haemophilia: A Case Report
}

\author{
Patrick Möhnle $^{a} \quad$ Isabell Pekrul ${ }^{\mathrm{a}} \quad$ Michael Spannagl $^{\mathrm{a}} \quad$ Andreas Sturm $^{\mathrm{b}}$ \\ Delila Singh $^{b}$ Claudia Dechant ${ }^{b}$ \\ ${ }^{a}$ Department of Transfusion Medicine, Cellular Therapeutics and Hemostaseology, Department of Anaesthesiology,

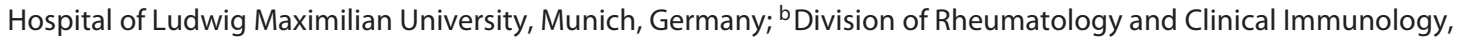 \\ Department of Internal Medicine IV, University of Munich, Munich, Germany
}

\section{Keywords}

Acquired haemophilia A · Antibodies monoclonal humanized · Drug therapy

\begin{abstract}
The prognosis of acquired haemophilia $\mathrm{A}(\mathrm{AHA})$ is severe and treatment options are limited. Emicizumab is a novel bispecific humanized monoclonal antibody in the treatment of inherited AHA with inhibitors. An 83-year-old AHA patient with congestive heart failure and a high risk for thromboembolic and cardiac events who had initially been treated successfully with steroids and substitution of recombinant Bdomain-deleted porcine FVIII developed severe bleeding complications and a secondary increase in inhibitor titres after 4 weeks of treatment. Conventional therapeutic strategies failed, and the patient was subsequently treated with emicizumab on off-label and named patient use premises. After the application of emicizumab, the clinical conditions stabilized and no further substitution of coagulation factors was needed. The patient could be discharged and survived 36 days in a cardiac rehabilitation centre without indications for spontaneous bleeding or thromboembolic events. We suggest that the effects of emicizumab in acquired haemophilia should be evaluated in clinical trials.
\end{abstract}

(c) 2019 S. Karger AG, Basel

P.M. and I.P. contributed equally and share first authorship.

\section{Introduction}

Treatment options in acquired haemophilia A (AHA) are limited and mainly based on anti-haemorrhagic management with bypassing agents or porcine factor VIII in combination with immunomodulation to eliminate autoantibodies to human coagulation factor VIII (h-FVIII). Anti-haemorrhagic treatment of AHA patients with activated recombinant factor VII ( $\mathrm{r}$-VIIa) is generally accepted as first-line therapy, activated prothrombin complex concentrate or recombinant porcine factor VIII (r p-FVIII) are alternative treatment options. The in-hospital mortality of the disease remains high despite the often intensive and costly therapeutic efforts [1]. We report an AHA patient with a severe form of acquired haemophilia showing an intractable bleeding phenotype who received off-label treatment with the novel bispecific monoclonal antibody emicizumab after conventional therapeutic strategies had failed.

\section{Case Report}

An 83-year-old male patient presented to our out-patient clinic with a history of spontaneous haematomas over the preceding 6 months. The patient had previously been diagnosed with congestive heart failure NYHA III, atrial fibrillation, chronic kidney disease stage 3, and had a history of repeated deep vein thrombosis and pulmonary embolism. Oral anticoagulation with phenprocoumon had been started 5 years ago and had been switched to a direct oral anticoagulant (DOAC) 9 months before. Laboratory assessment proved the diagnosis of AHA with a factor VIII activity of $<1 \%$ and a high titre of inhibitory antibodies (97 Bethesda units;

\section{KARGER}

(C) 2019 S. Karger AG, Basel 
BU). Clinical suspicion of acute bleeding anaemia with signs of dyspnoea, tachycardia, hypotension, and weakness was supported by a haemoglobin level of $6.8 \mathrm{~g} / \mathrm{dL}$. Oral anticoagulation was stopped and the patient was admitted for in-patient treatment. Due to the patient's high thromboembolic and cardiovascular risk and active bleeding signs with a potential need of surgical intervention, the bypassing agents r-VIIa and activated prothrombin complex concentrate were considered to promote an incalculably high risk of thromboembolic complications with limited laboratory monitoring options. We therefore decided to achieve normalization of factor VIII activity by substitution of B-domain-deleted r p-FVIII with factor VIII activity peak level targets of $80-100 \%$ [2]. A sufficient factor VIII recovery was confirmed $3 \mathrm{~h}$ after the first bolus injection of $\mathrm{r}$ p-FVIII, followed by a modified Bethesda assay showing negligible cross-reactivity of anti-FVIII autoantibodies to $\mathrm{r}$ p-FVIII. Gastroscopy was successfully performed to treat gastric angiodysplasia as a source of gastric bleeding. After sustained bleeding control was achieved the $\mathrm{r}$ p-FVIII doses were decreased to approximately $50 \mathrm{IU} / \mathrm{kg}$ body weight (BW) twice a day with a factor VIII activity peak level target of 40-60\% [3]. For immunomodulation the patient was started on prednisolone (initial dose $1 \mathrm{mg} / \mathrm{kg} \mathrm{BW}$ once daily). During the first treatment weeks we ruled out specific AHA aetiologies via laboratory work-up and computed tomography. Overall, this treatment approach showed to be successful in the first 3 weeks. No further active bleedings were detected, the haemoglobin levels remained stable without red blood cell concentrate (RBCC) transfusion requirements, and the inhibitor titre declined to $12 \mathrm{BU}$. The significant and satisfactory clinical improvement led us to the decision to support the patient's wish for a temporary discharge over the Christmas holidays with temporary discontinuation of the $\mathrm{r}$ p-FVIII treatment.

However, the patient was readmitted after 6 days with increasing clinical bleeding signs including musculus biceps femoris haematoma. With continuation of $r \mathrm{p}$-FVIII substitution the progression of haematoma initially stopped.

However, 5 days later after a scheduled colonoscopy the patient's condition deteriorated with signs of sepsis and decompensated congestive heart failure as well as severe haemorrhage signs (several new large haematomas, prolonged bleeding from the ear lobe after capillary blood collection) with the need for transfusion of 6-8 RBCC over 1 week. Blood culture revealed Morganella morganii as a cause of sepsis.

Concomitantly, a dramatic rise in inhibitory titres to h-FVIII (236 BU) and especially to $\mathrm{r}$ p-FVIII (31 BU) was observed, preventing further substitution therapy with $\mathrm{r}$ p-FVIII. Anti-haemorrhagic therapy was switched to r-VIIa with doses up to $10 \mathrm{mg}$ every $4 \mathrm{~h}$ due to the exacerbating high clinical bleeding disposition. Additionally, the replacement therapy had to be supplemented with prothrombin complex concentrate, factor XIII, and fibrinogen, as well as transfusion of 6-8 units of RBCCs per week.

After the start of a specific antimicrobial treatment immunomodulation was extended by rituximab ( 4 weekly doses of $375 \mathrm{mg} /$ $\mathrm{m}^{2}$ body surface area) and high-dose intravenous immunoglobulins $[4,5]$. Plasmapheresis was discussed as a therapeutic option but could not be performed due to the patient's unstable condition and insufficient possibilities of vascular access.

Despite recovery from the systemic infection and decline of the inhibitory antibody titre upon this maximal therapy, during the following weeks the patient continued to develop large diffuse haematomas involving the torso, musculus iliopsoas, and extremities, partly with compartment syndrome followed by nerve lesions. Moreover, several small and otherwise irrelevant skin lesions led to severe bleeding complications despite additional local treatment with haemostyptic and other adjuvants. The patient was rendered immobile and bedridden as a result of massive swelling and pain of the legs and arms, partly because of haematomas and the concomitant deterioration of congestive heart failure with decreasing renal and hepatic function. In light of the ongoing life-threatening condition of the patient, further waiting for remission after steroid/rituximab immunosuppression or administration of additional immunosuppressive agents was not an option, since data from clinical trial results indicate a prolonged time to remission for this immunosuppressant therapy regime $[4,6]$. Despite the seriousness of the disease the patient had an unaltered mental status and repeatedly expressed the will to live.

\section{Discussion}

The novel therapeutic compound emicizumab, a newly developed humanized bispecific monoclonal antibody to factors IXa and X for the treatment of inherited haemophilia with inhibitors, recently proved to be highly effective and received FDA approval in the USA $[7,8]$. Due to the similarity of the disease mechanisms with alloantibodies to factor VIII in congenital haemophilia with inhibitors, and autoantibodies in AHA, similar effects of the compound in both disease entities must be expected [9, 10]. Following extensive interdisciplinary discussion and evaluation of therapeutic options, rapid disease progression, and the patient's will, taking into account our clinical and laboratory expertise with the new compound, we unequivocally consented to administer emicizumab as an ultima ratio therapy under the local regulatory conditions of off-label use.

Our therapy goals were: (1) reduction of bleeding, and (2) sufficient wound healing with the lowest possible effective emicizumab drug level with regard to the thromboembolic risk factors. Under continuous monitoring of vital signs and close clinical surveillance, we administered the first subcutaneous injection of $3 \mathrm{mg} /$ $\mathrm{kg}$ BW emicizumab. Concomitantly we prolonged the r-VIIa dosing intervals to every $8 \mathrm{~h}$ for the first $24 \mathrm{~h}$. No additional blood or plasma products were required. After detecting emicizumab in the patient's plasma with a newly developed clotting test calibrated for emicizumab drug monitoring, r-VIIa intervals were reduced to every $12 \mathrm{~h}$ and stopped 2 days after the first emicizumab injection. Simultaneously, a markedly and rapid overall clinical improvement was noted, no further spontaneous bleeding events occurred, and fast haematoma resorption was observed. Cardiac recompensation was achieved within 2 weeks after the first injection. With physical therapy the patient regained physical strength and ambulation was possible. After the first emicizumab injection maximum drug levels of $13 \mu \mathrm{g} /$ $\mathrm{mL}$ were detected. After a second emicizumab injection (1.5 mg/kg BW, 7 days after first dosing), we achieved maximum drug levels of $33 \mu \mathrm{g} / \mathrm{mL}$. Considering the overall clinical picture, we decided to maintain a drug 
level of $20-30 \mu \mathrm{g} / \mathrm{mL}$. The third and last injection of emicizumab $(1.5 \mathrm{mg} / \mathrm{kg}$ BW, 20 days after first administration) was administered on the day of hospital discharge.

After 1 week in a domestic environment without the requirement of nursing services, the patient started an inpatient follow-up treatment at a cardiac rehabilitation centre. For follow-up, we regularly visited the patient in the rehabilitation centre and performed measurements of anti-h-FVIII inhibitor titres (16 BU on day of discharge), emicizumab drug levels $(27 \mu \mathrm{g} / \mathrm{mL})$, and FVIII chromogenic (bovine, to detect the recurrence of endogenic FVIII, 4\%) assays. Clinically, a steady improvement was observed over the course of weeks, with the patient able to go on regular walks outside the rehabilitation centre. Emicizumab levels steadily declined to $21 \mu \mathrm{g} / \mathrm{mL} 5$ weeks after the last administration with anti-h-FVIII inhibitor titres of 2 BU and an endogenous FVIII activity of $10 \%$ in bovine chromogenic FVIII assay. However, 36 days after hospital discharge and the last emicizumab dosing, the patient passed away while sleeping. With no clinical or laboratory signs of bleeding or thromboembolic events (declining D-dimer levels over the clinical course) and no autopsy performed, an arrhythmic event was regarded as the most probable cause of death by the treating physicians.

Although unlikely, thromboembolic events as well as bleeding possibly related to AHA as well as to medication cannot be ruled out completely as the cause of death. Still, in this individual case, the application of emicizumab as an ultima ratio therapy led to a rapid and dramatic short- term clinical improvement in an otherwise moribund patient. We propose to evaluate the use of emicizumab in AHA in clinical trials.

\section{Acknowledgements}

We wish to thank the team of Roche Germany for organising the delivery of the first dose of the drug emicizumab via international pharmacy according to paragraph 73.3 of German drug law.

\section{Statement of Ethics}

We thank our patient and his widow for allowing his data to be presented.

\section{Disclosure Statement}

P.M. served on an advisory board for CSL Behring, received lecture fees from CSL Behring and Roche, and grants from Shire, Pfizer, and Biotest. I.P. received lecture fees from Sobi and grants form LFB and Novo Nordisk. M.S. served on the advisory board for Roche, Bayer, SOBI, Pfizer, CSL Behring, Novo Nordisc, and Shire, and received research grants from Pfizer, Shire, and SOBI. C.D. served on advisory boards for Roche.

\section{Author Contributions}

P.M., I.P., M.S., D.S., A.S., and C.D. coordinated the patient care and collected and analysed data. I.P. established laboratory monitoring methods under emicizumab therapy. P.M. and I.P. wrote the report. All authors contributed to the content of the report.

\section{References}

1 Knoebl P, Marco P, Baudo F, Collins P, HuthKühne A, Nemes L, et al.; EACH2 Registry Contributors. Demographic and clinical data in acquired hemophilia A: results from the European Acquired Haemophilia Registry (EACH2). J Thromb Haemost. 2012 Apr; 10(4):622-31.

2 Kruse-Jarres R, St-Louis J, Greist A, Shapiro A, Smith H, Chowdary P, et al. Efficacy and safety of OBI-1, an antihaemophilic factor VIII (recombinant), porcine sequence, in subjects with acquired haemophilia A. Haemophilia. 2015 Mar;21(2):162-70.

3 Stemberger M, Möhnle P, Tschöp J, Ney L, Spannagl M, Reincke M. Successful bleeding control with recombinant porcine factor VIII in reduced loading doses in two patients with acquired haemophilia A and failure of bypassing agent therapy. Haemophilia. 2016 Sep; 22(5):e472-4.
4 Collins P, Baudo F, Knoebl P, Lévesque H, Nemes L, Pellegrini F, et al.; EACH2 registry collaborators. Immunosuppression for acquired hemophilia $\mathrm{A}$ : results from the European Acquired Haemophilia Registry (EACH2). Blood. 2012 Jul;120(1):47-55.

5 Zeitler H, Ulrich-Merzenich G, Hess L, Konsek E, Unkrig C, Walger P, et al. Treatment of acquired hemophilia by the Bonn-Malmo Protocol: documentation of an in vivo immunomodulating concept. Blood. 2005 Mar; 105(6):2287-93.

6 Tiede A, Klamroth R, Scharf RE, Trappe RU, Holstein K, Huth-Kühne A, et al. Prognostic factors for remission of and survival in acquired hemophilia $\mathrm{A}$ (AHA): results from the GTH-AH 01/2010 study. Blood. 2015 Feb; 125(7):1091-7.
7 Sampei Z, Igawa T, Soeda T, Okuyama-Nishida Y, Moriyama C, Wakabayashi T, et al. Identification and multidimensional optimization of an asymmetric bispecific IgG antibody mimicking the function of factor VIII cofactor activity. PLoS One. 2013;8(2):e57479.

8 Oldenburg J, Mahlangu JN, Kim B, Schmitt C, Callaghan MU, Young G, et al. Emicizumab Prophylaxis in Hemophilia A with Inhibitors. N Engl J Med. 2017 Aug;377(9):809-18.

9 Muto A, Yoshihashi K, Takeda M, Kitazawa T, Soeda T, Igawa T, et al. Anti-factor IXa/X bispecific antibody ACE910 prevents joint bleeds in a long-term primate model of acquired hemophilia A. Blood. 2014 Nov; 124(20):3165-71.

10 Takeyama N. New option of hemostatic treatment for aquired hemophilia A by single injection of emicizumab, FVIIIa mimicking bispecific antibody, irrespective of eptiope specificity [abstract M-P-002 (471)]. Haemophilia. 2018;24:12. 